\title{
Autoantibodies to N-terminally truncated GAD improve clinical phenotyping of individuals with adult-onset diabetes: Action LADA 12
}

\author{
Peter Achenbach ${ }^{1,2,3,4}$ (D) Mohammed I. Hawa ${ }^{5} \cdot$ Stephanie Krause $^{1,2}$ • Vito Lampasona ${ }^{6} \cdot$ Samuel T. Jerram $^{5}$. \\ Alistair J. K. Williams ${ }^{7} \cdot$ Ezio Bonifacio $^{4,8,9} \cdot$ Anette G. Ziegler $^{1,2,3,4} \cdot$ R. David Leslie $^{5}$ - on behalf of the Action LADA \\ consortium
}

Received: 30 December 2017 / Accepted: 8 March 2018 / Published online: 4 April 2018

(C) The Author(s) 2018

\begin{abstract}
Aims/hypothesis Adult-onset type 1 diabetes, in which the $65 \mathrm{kDa}$ isoform of GAD (GAD65) is a major autoantigen, has a broad clinical phenotype encompassing variable need for insulin therapy. This study aimed to evaluate whether autoantibodies against N-terminally truncated GAD65 more closely defined a type 1 diabetes phenotype associated with insulin therapy.

Methods Of 1114 participants with adult-onset diabetes from the Action LADA (latent autoimmune diabetes in adults) study with sufficient sera, we selected those designated type $1(n=511)$ or type 2 diabetes $(n=603)$ and retested the samples in radiobinding assays for human full-length GAD65 autoantibodies (f-GADA) and N-terminally truncated (amino acids 96585) GAD65 autoantibodies (t-GADA). Individuals' clinical phenotypes were analysed according to antibody binding patterns. Results Overall, 478 individuals were f-GADA-positive, 431 were t-GADA-positive and 628 were negative in both assays. Risk of insulin treatment was augmented in t-GADA-positive individuals (OR 4.69 [95\% CI 3.57, 6.17]) compared with f-GADApositive individuals (OR 3.86 [95\% CI 2.95, 5.06]), irrespective of diabetes duration. Of 55 individuals who were f-GADApositive but t-GADA-negative, i.e. with antibody binding restricted to the N-terminus of GAD65, the phenotype was similar to type 2 diabetes with low risk of progression to insulin treatment. Compared with these individuals with N-terminal GAD65restricted GADA, t-GADA-positive individuals were younger at diagnosis $(p=0.005)$, leaner $(p<0.0001)$ and more often had multiple diabetes-associated autoantibodies $(28.3 \%$ vs $7.3 \% ; p=0.0005)$.

Conclusions/interpretation In individuals with adult-onset diabetes, presence of N-terminally truncated GAD65 autoantibodies is associated with the clinical phenotype of autoimmune type 1 diabetes and predicts insulin therapy.
\end{abstract}

A list of members of the Action LADA consortium is available in the Appendix.

Electronic supplementary material The online version of this article (https://doi.org/10.1007/s00125-018-4605-3) contains peer-reviewed but unedited supplementary material, which is available to authorised users.

Peter Achenbach

peter.achenbach@helmholtz-muenchen.de

R. David Leslie

r.d.g.leslie@qmul.ac.uk

1 Institute of Diabetes Research, Helmholtz Zentrum München, German Research Center for Environmental Health, Ingolstaedter Landstrasse 1, 85764 Munich-Neuherberg, Germany

2 Forschergruppe Diabetes, Technische Universität München, at Klinikum rechts der Isar, Munich, Germany

3 German Center for Diabetes Research (DZD), Munich-Neuherberg, Germany

4 Forschergruppe Diabetes e.V. at Helmholtz Zentrum München, German Research Center for Environmental Health, Munich-Neuherberg, Germany
5 Centre for Immunobiology, Blizard Institute, Barts and the London School of Medicine and Dentistry, Queen Mary University of London, London E1 2AT, UK

6 Division of Genetics and Cell Biology, San Raffaele Scientific Institute, Milan, Italy

7 Diabetes and Metabolism, School of Clinical Sciences, Southmead Hospital, University of Bristol, Bristol, UK

8 DFG Research Center for Regenerative Therapies Dresden, Faculty of Medicine, Technische Universität Dresden, Dresden, Germany

9 Paul Langerhans Institute Dresden, German Center for Diabetes Research (DZD), Technische Universität Dresden, Dresden, Germany 


\section{Research in context}

\section{What is already known about this subject?}

- Defining the type of diabetes based on disease aetiology in individuals with adult-onset diabetes is problematic

- Autoantibodies against the $65 \mathrm{kDa}$ isoform of GAD (GAD65) are used as a diagnostic immune biomarker of adult-onset type 1 diabetes

- The clinical phenotype varies among GAD65 autoantibody (GADA)-positive adult diabetic individuals with variable need for insulin therapy

\section{What is the key question?}

- Do autoantibodies against N-terminally truncated GAD65 more closely define adult-onset type 1 diabetes and better predict a requirement for insulin therapy?

\section{What are the new findings?}

- Using sera of 1114 individuals with adult-onset diabetes, we show that the presence of autoantibodies against Nterminally truncated (aa 96-585) GAD65 is associated with the clinical phenotype of type 1 diabetes and an increased need for insulin therapy

- In contrast, the presence of GADA with binding restricted to N-terminal GAD65 epitopes is associated with a similar clinical phenotype to GADA-negative, type 2 diabetic individuals

\section{How might this impact on clinical practice in the foreseeable future?}

- Screening for N-terminally truncated GAD65 autoantibodies improves the clinical phenotyping of individuals with adult-onset diabetes. This might have important practical implications given the relatively large number of adults who are screened for GADA, both for diabetes classification and prediction of risk for insulin therapy

Keywords Adult-onset diabetes $\cdot$ Autoantibodies $\cdot$ Autoimmune $\cdot$ Clinical phenotype $\cdot$ GAD $\cdot$ LADA $\cdot$ N-terminally truncated GAD65 · Patients · Type 1 diabetes

$\begin{array}{ll}\text { Abbreviations } \\ \text { aa } & \text { Amino acids } \\ \text { GAD65 } & \text { 65 kDa isoform of GAD } \\ \text { GADA } & \text { GAD autoantibodies } \\ \text { f-GADA } & \text { Full-length (aa 1-585) GAD65 autoantibodies } \\ \text { t-GADA } & \text { N-terminally truncated (aa 96-585) } \\ & \text { GAD65 autoantibodies } \\ \text { IA-2A } & \text { Insulinoma-associated antigen-2 autoantibodies } \\ \text { IQR } & \text { Interquartile range } \\ \text { LADA } & \text { Latent autoimmune diabetes in adults } \\ \text { NIDDK } & \text { National Institute of Diabetes and Digestive } \\ \text { ZnT8A } & \begin{array}{l}\text { and Kidney diseases } \\ \text { Zinc transporter-8 autoantibodies }\end{array}\end{array}$

\section{Introduction}

Type 1 diabetes can occur at any age and is of variable severity. In adults presenting with the disease, unlike children, it may masquerade as type 2 diabetes, since insulin therapy is not always required [1]. Established autoimmune markers of type 1 diabetes, notably GAD autoantibodies (GADA), have been used to screen individuals presenting with adult-onset diabetes to identify those with type 1 diabetes, including the so-called latent autoimmune diabetes in adults (LADA) [2, 3]. Identifying individuals with adult-onset type 1 diabetes is important as, compared with people with type 2 diabetes, they have a different clinical course and different comorbidities [1]. In adult-onset diabetes, the detection of GADA together with insulinoma-associated antigen-2 autoantibodies (IA-2A) or zinc transporter-8 autoantibodies (ZnT8A) is highly predictive of the need for insulin therapy [4]. Nevertheless, the majority of antibody-positive individuals with adult-onset diabetes present with GADA alone, which may represent either falsepositive antibody assay signals in an adult population with a relatively low prevalence of autoimmune type 1 diabetes, or true-positive autoantibodies with high risk for progression to insulin therapy. Antibody characteristics might facilitate diagnosis and estimation of risk for disease progression because high-affinity GADA responses against the middle (amino acids [aa] 235-444) and C-terminal (aa 445-585) domains of the $65 \mathrm{kDa}$ isoform of GAD (GAD65) were associated with type 1 diabetes development in children and adolescents [5]; and high antibody titre [6] and binding to C-terminal (aa 437585) GAD65-depending epitopes were reported as characteristic features of LADA individuals [7]. In contrast, low- 
affinity GADA and/or antibody responses restricted to the Nterminal (aa 1-95) domain of GAD65 were not associated with classic type 1 diabetes [5, 8]. Furthermore, using Nterminally truncated (aa 96-585) GAD65 antigen in GADA radiobinding assays improved the specificity for type 1 diabetes [9]. The aim of this study was to evaluate N-terminally truncated (aa 96-585) GAD65 autoantibodies (t-GADA), compared with full-length GAD65 autoantibodies (fGADA), in a large cohort of adult-onset diabetes individuals with variable clinical phenotype.

\section{Methods}

Individuals and samples Sera were obtained from individuals with adult-onset diabetes participating in the Action LADA study [3]. The study design is cross-sectional and includes individuals with adult-onset diabetes (originally $n=6156$; aged 30-70 years; diabetes diagnosed within 5 years) with the aim of identifying risk factors for adult-onset autoimmune diabetes (www.actionlada.org). The study protocol is in accordance with the Declaration of Helsinki and was approved by local ethics committees. Informed written consent was obtained from all individuals before blood sampling. The study was approved by the UK National Research Ethics Committee (no. P/02/240).

All samples had previously been tested for GADA, IA-2A and $\mathrm{ZnT} 8 \mathrm{~A}$ in the central laboratory in London [3]. For the current study, we selected two groups from our initial cohort: of those with sufficient sera in storage, 511 individuals with beta cell autoantibodies (all GADA-positive), and 603 individuals without beta cell autoantibodies of similar age and diabetes duration (Table 1). Of these 1114 participants, the median age at diagnosis was 48.2 years (interquartile range [IQR ] 39.5-56.3 years) and median disease duration at sample collection was 2.0 years (IQR 0.6-3.5 years); while information on diabetes treatment and BMI was available for 1045 (94\%) and 1059 (95\%) individuals, respectively.

Antibody measurements Sera of the 1114 participants were retested for GADA in the Munich laboratory using the National Institute of Diabetes and Digestive and Kidney diseases (NIDDK) harmonised radiobinding assay [10]. In vitro transcribed/translated and $\left[{ }^{35} \mathrm{~S}\right]$-labelled human full-length (aa 1-585) GAD65 and N-terminally truncated (aa 96-585) GAD65 (cloned by Vito Lampasona, San Raffaele Institute, Milan, Italy) were used as antigens to detect f-GADA and tGADA, respectively. The assays achieved adjusted sensitivities of $64 \%$ (f-GADA) and $72 \%$ (t-GADA) at $95 \%$ specificity in the 2012 Islet Autoantibody Standardization Program workshop [9]. Throughout all measurements, investigators were blinded with respect to the previously determined autoantibody status of the samples.
Statistical analysis We used the Mann-Whitney $U$ test to compare continuous variables, Fisher's exact test to compare frequencies and Spearman correlation to determine the correlation between antibody titres. Data are presented, where appropriate, as medians with IQRs or ORs with $95 \%$ CIs. For all analyses, a two-tailed $p$ value of $<0.05$ was considered significant. Statistical analyses were performed using the GraphPad Prism 3 program (GraphPad Software, La Jolla, CA, USA).

\section{Results}

Of 1114 participants with adult-onset diabetes, 478 were fGADA-positive using the NIDDK harmonised radiobinding assay. Of these 478 f-GADA-positive individuals, 55 (11.5\%) were t-GADA-negative, thus demonstrating antibody binding restricted to N-terminal GAD65 epitopes (Fig. 1a). Such restricted responses were seen in four $(3.2 \%)$ of the $126 \mathrm{f}$ GADA-positive individuals who also had IA-2A and/or ZnT8A (electronic supplementary material [ESM] Fig. 1a), in contrast to $51(14.5 \%)$ of the 352 individuals who only had f-GADA positivity (ESM Fig. 1b; $p=0.0003$ ). Overall, 431 individuals were t-GADA-positive. Antibody titres were strongly correlated between f-GADA and t-GADA assays $(r=0.86, p<0.0001)$.

For clinical phenotype, f-GADA-positive individuals were diagnosed with diabetes at a median age of 47.0 years (IQR $38.4-55.7$ years), median BMI of $25.6 \mathrm{~kg} / \mathrm{m}^{2}$ (IQR 22.8 $29.4 \mathrm{~kg} / \mathrm{m}^{2}$ ) and $51.2 \%$ required insulin therapy (Table 1 ). For the t-GADA-positive individuals, median age at diagnosis was 46.6 years (IQR 38.0-55.5 years), median BMI $24.9 \mathrm{~kg}$ / $\mathrm{m}^{2}$ (IQR $22.6-28.8 \mathrm{~kg} / \mathrm{m}^{2}$ ) and $55.3 \%$ were on insulin therapy. For the whole cohort, risk of insulin treatment was augmented in t-GADA-positive individuals (OR 4.69 [95\% CI $3.57,6.17] ; p<0.0001$ vs t-GADA-negative) compared with f-GADA-positive individuals (OR 3.86 [95\% CI 2.95, 5.06]; $p<0.0001$ vs f-GADA-negative). This difference was evident irrespective of the disease duration: both for the cohorts assessed at 1 month post-diagnosis (OR 5.20 [95\% CI 3.89, 6.94] and 4.42 [3.32, 5.88], respectively) and at 6 months post-diagnosis (OR 4.92 [95\% CI 3.61, 6.69] and 4.25 [95\% CI 3.13, 5.77], respectively).

Strikingly, when comparing the t-GADA-positive individuals with individuals who were N-terminal GAD65-restricted in antibody binding, i.e. f-GADA-positive but t-GADA-negative, the latter required insulin treatment less often $(13.7 \%$; $p<0.0001$; Fig. 1b), were older at diagnosis (median age 52.0 years [IQR 41.8-59.8 years]; $p=0.005$; Fig. $1 \mathrm{c}$ ) and were more obese (median BMI $29.9 \mathrm{~kg} / \mathrm{m}^{2}$ [IQR 26.7-35.9 kg/m²]; $p<0.0001$; Fig. 1d). Moreover, individuals with N-terminal GAD65-restricted antibodies were not phenotypically different from individuals who were negative for both f-GADA and tGADA, i.e. type 2 diabetes individuals (Fig. 1, Table 1). 


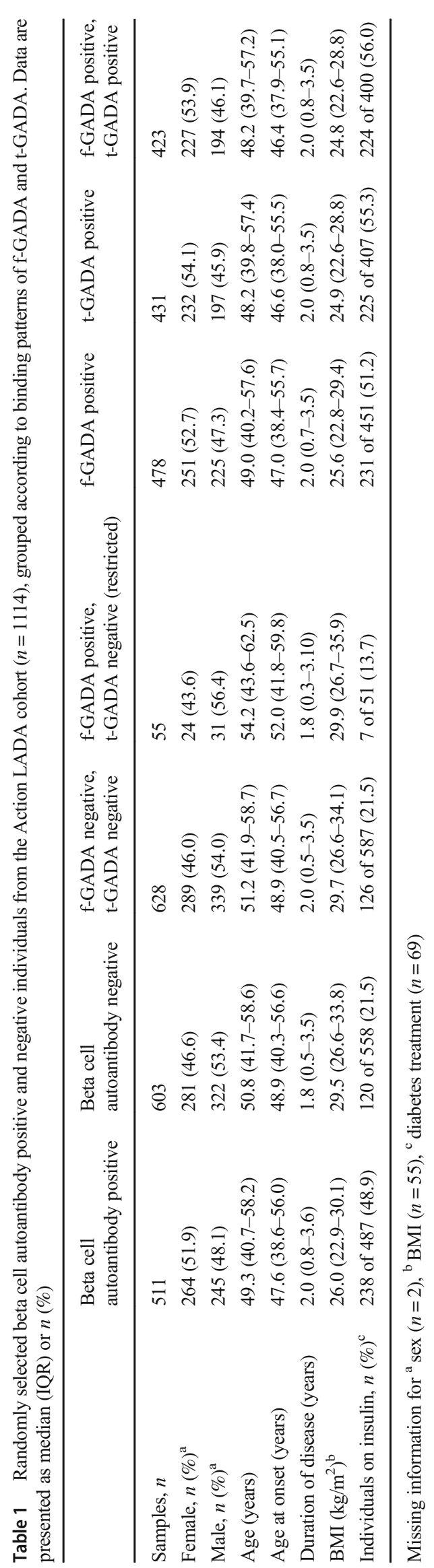


Fig. 1 Relationship between GADA epitope reactivity and clinical phenotype. In (a), levels of f-GADA ( $x$-axis) are plotted against levels of t-GADA ( $y$-axis) using $\log _{2}$ scales for 1114 participants with adult-onset diabetes. Dashed lines indicate thresholds for positivity for $\mathrm{f}$ GADA (35 units) and t-GADA (25 units). In (b-d), individuals are grouped according to positive or negative status of f-GADA and t-GADA, and compared with respect to frequency of insulin treatment (b), age at diagnosis (c) and BMI (d). Median (IQR) values are shown for each group in (c) and (d). $* * p<0.01$, $* * * p<0.001$
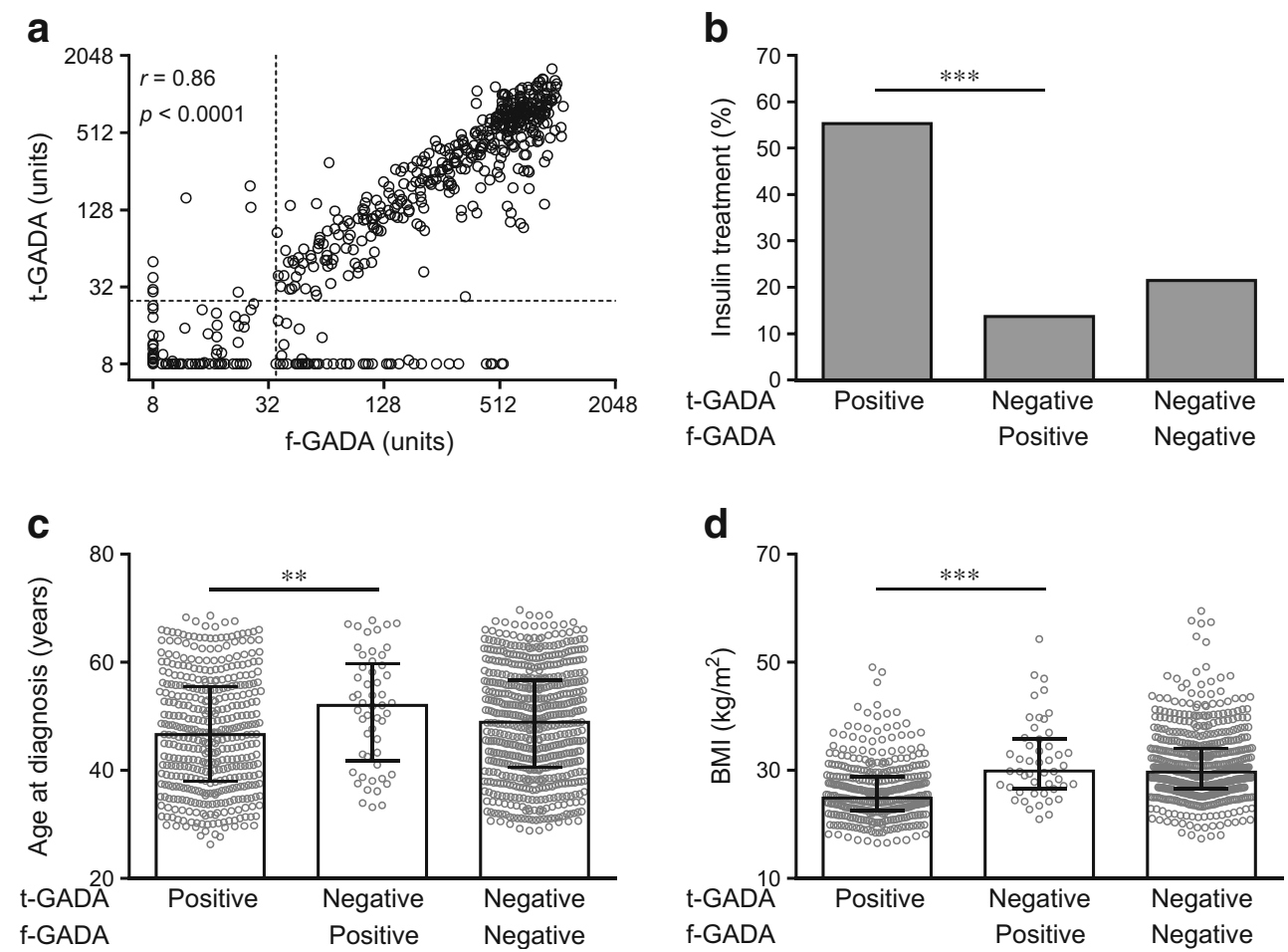

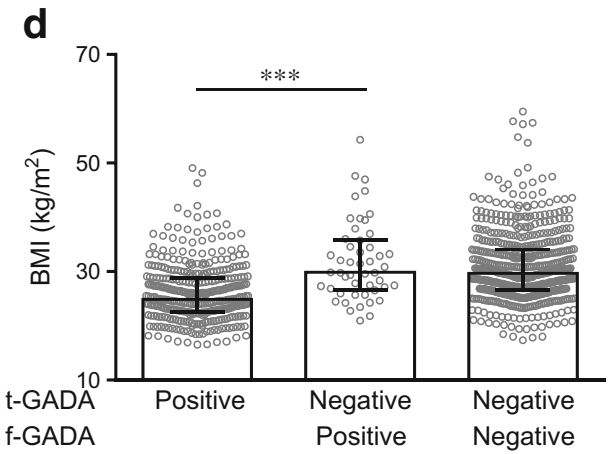

Finally, when comparing the individuals who were positive $(n=423)$ or negative $(n=628)$ in both assays (f-GADA and tGADA), these positive individuals were younger at diagnosis $(p=0.003)$, leaner $(p<0.0001)$ and were more often on insulin treatment $(p<0.0001)$ (Table 1$)$.

\section{Discussion}

In this study, we found that overall, f-GADA-positive participants and t-GADA-positive participants with adult-onset diabetes had a similar clinical phenotype that distinguished them from GADA-negative participants with type 2 diabetes. Importantly, however, a subgroup of individuals who were positive for f-GADA but negative for t-GADA (i.e. individuals with $\mathrm{N}$-terminal GAD65-restricted GADA) had a similar clinical phenotype to GADA-negative, type 2 diabetic individuals, albeit in relatively low numbers. It is therefore possible that the N-terminal GAD65-restricted GADA, as proposed, does not detect individuals with autoimmune type 1 diabetes, at least using clinical phenotype as a proxy, whilst screening for t-GADA could be helpful for clinical assessment and prediction of insulin therapy.

Screening adult-onset diabetes individuals for GADA to identify autoimmune type 1 diabetes is limited by falsepositive and false-negative assay results [1]. Using the tGADA assay, when compared with the f-GADA assay, we identified a reduced number of GADA-positive individuals $(88 \%)$ and confirmed a high number $(99 \%)$ of GADAnegative individuals. Further, we found that the risk of being on insulin treatment within 5 years from diagnosis when positive for t-GADA was higher (OR 4.69) than in those individuals positive for f-GADA (OR 3.86). Those positive for tGADA, compared with those with N-terminal GAD65-restricted GADA, were enriched for phenotypic features characteristic of individuals with adult-onset type 1 diabetes, including being younger, leaner and with an increased need for insulin treatment.

Differences in GADA epitope binding between LADA individuals with clinical characteristics similar to either type 1 or type 2 diabetes have previously been reported [7]. Individuals with antibodies against epitopes within the $\mathrm{N}$-terminal/middle (aa 1-360) GAD65 region and negative for antibodies against the C-terminal (aa 437-585) GAD65 region showed clinical features that were indistinguishable from those of GADAnegative individuals, whereas the presence of autoantibodies directed to both GAD65 regions identified a subgroup of individuals with low BMI, low basal C-peptide values and the need for insulin treatment [7]. In contrast to this former study and based on our previous data [5,9], we used the Nterminally truncated (aa 96-585) GAD65 antigen for our tGADA assay, containing epitopes within the middle and Cterminal GAD65 regions. We believe that the false-positive rate in screening tests can likely be decreased by using this t-GADA assay to identify those at risk of progression to insulin treatment.

In conclusion, our findings suggest that in individuals with adult-onset diabetes, screening for t-GADA augments the clinical phenotype of autoimmune type 1 diabetes. This may have important practical implications given the relatively large 
number of adult patients who are screened for GADA, both for diabetes classification and prediction of risk for insulin therapy.

Data availability The datasets generated and/or analysed during the current study are available from the corresponding author on reasonable request.

Funding This study was supported in part by grants from the German Federal Ministry of Education and Research (BMBF) to the Competence Network for Diabetes mellitus (FKZ 01GI0805) and the German Center for Diabetes Research (DZD e.V.), and from JDRF (2-SRA-2015-50-Q$\mathrm{R})$. The Action LADA study was supported by the 5th Framework Programme of the European Union. EB is supported by the DFG Research Center and Cluster of Excellence - Center for Regenerative Therapies Dresden (FZ 111).

Duality of interest The authors declare that there is no duality of interest associated with this manuscript.

Contribution statement PA and RDL designed and coordinated the study, researched data, and wrote, reviewed and edited the manuscript. $\mathrm{MIH}$ contributed to acquisition, analysis and interpretation of data, and reviewed and edited the manuscript. SK performed GADA measurements, researched data, contributed to the discussion, and drafted, reviewed and edited the manuscript. VL, STJ, AJKW, EB and AGZ contributed to the analysis, interpretation of data and discussion, and reviewed and edited the manuscript. VL, AJKW and PA developed the concept of screening for autoantibodies against N-terminally truncated GAD65 for improved type 1 diabetes specificity. All listed authors approved the final version of the manuscript. PA is the guarantor of this work and, as such, had full access to all the data in the study and takes responsibility for the integrity of the data and the accuracy of the data analysis.

\section{Appendix: Members of the Action LADA consortium}

Richard David Leslie and Mohammed I Hawa, Blizard Institute, Queen Mary University of London, London, UK

Paolo Pozzilli, University Campus Bio-Medico, Rome, Italy

Henning Beck-Nielsen and Knud Yderstraede, University Hospital of Odense, Odense, Denmark

Steven Hunter and David Hadden, Royal Victoria Hospital, Belfast, UK

Raffaella Buzzetti, University La Sapienza, University of Rome, Rome, Italy

Werner Scherbaum and Hubert Kolb, University of Düsseldorf, Düsseldorf, Germany

Nanette C. Schloot, German Diabetes Centre, University of Düsseldorf, Düsseldorf and Clinic for Metabolic Diseases at University Hospital Düsseldorf, Germany. N.C. Schloot is guest scientist at the German Diabetes Center, Institute of Clinical Diabetology, Düsseldorf and currently employed by Lilly Deutschland, Bad Homburg, Germany

Jochen Seissler, Ludwig-Maximilians-University, Munich, Germany
Guntram Schernthaner, Rudolfstiftung Hospital, Vienna, Austria

Jaakko Tuomilehto and Cinzia Sarti, National Institute for Health and Welfare, Helsinki, Finland

Alberto De Leiva and Eulalia Brugues, Universitat Autonoma de Barcelona, Barcelona, Spain

Didac Mauricio, University Hospital Germans Trias Pujol, Badalona, Spain

Charles Thivolet, Hopital Edouard Herriot, Lyon, France

Open Access This article is distributed under the terms of the Creative Commons Attribution 4.0 International License (http://creativecommons. org/licenses/by/4.0/), which permits unrestricted use, distribution, and reproduction in any medium, provided you give appropriate credit to the original author(s) and the source, provide a link to the Creative Commons license, and indicate if changes were made.

\section{References}

1. Leslie RD, Palmer J, Schloot NC, Lernmark A (2016) Diabetes at the crossroads: relevance of disease classification to pathophysiology and treatment. Diabetologia 59:13-20

2. Tuomi T, Groop LC, Zimmet PZ, Rowley MJ, Knowles W, Mackay IR (1993) Antibodies to glutamic acid decarboxylase reveal latent autoimmune diabetes mellitus in adults with a non-insulindependent onset of disease. Diabetes 42:359-362

3. Hawa MI, Kolb H, Schloot N et al (2013) Adult-onset autoimmune diabetes in Europe is prevalent with a broad clinical phenotype: Action LADA 7. Diabetes Care 36:908-913

4. Lampasona V, Petrone A, Tiberti $\mathrm{C}$ et al (2010) Zinc transporter 8 antibodies complement GAD and IA-2 antibodies in the identification and characterization of adult-onset autoimmune diabetes: non insulin requiring autoimmune diabetes (NIRAD) 4. Diabetes Care 33:104-108

5. Mayr A, Schlosser M, Grober N et al (2007) GAD autoantibody affinity and epitope specificity identify distinct immunization profiles in children at risk for type 1 diabetes. Diabetes 56:1527-1533

6. Buzzetti R, Di Pietro S, Giaccari A et al (2007) High titer of autoantibodies to GAD identifies a specific phenotype of adult-onset autoimmune diabetes. Diabetes Care 30:932-938

7. Falorni A, Gambelunghe G, Forini F et al (2000) Autoantibody recognition of $\mathrm{COOH}$-terminal epitopes of GAD65 marks the risk for insulin requirement in adult-onset diabetes mellitus. J Clin Endocrinol Metab 85:309-316

8. Krause S, Landherr U, Agardh CD et al (2014) GAD autoantibody affinity in adult patients with latent autoimmune diabetes, the study participants of a GAD65 vaccination trial. Diabetes Care 37:16751680

9. Williams AJ, Lampasona V, Schlosser M et al (2015) Detection of antibodies directed to the $\mathrm{N}$-terminal region of GAD is dependent on assay format and contributes to differences in the specificity of GAD autoantibody assays for type 1 diabetes. Diabetes 64:32393246

10. Bonifacio E, Yu L, Williams AK et al (2010) Harmonization of glutamic acid decarboxylase and islet antigen-2 autoantibody assays for national institute of diabetes and digestive and kidney diseases consortia. J Clin Endocrinol Metab 95:3360-3367 